\title{
Potential Association between Anterior Cruciate Ligament Tear and "Bi-Collateral" Ligamentous Rupture: A Retrospective Study
}

\author{
Jean Roger Moulion Tapouh ${ }^{*}$, Boniface Moifo ${ }^{1,2}$, Cathy Monabang Zoé1,3, \\ Marc Leroy Guifo ${ }^{3,4}$, Haoua Tebere1, Annick Laure Edzimbi', Samuel Nko'o Amvene ${ }^{1,3}$ \\ ${ }^{1}$ Department of Radiology and Radiation Oncology, Faculty of Medicine and Biomedical Sciences (FMBS), \\ The University of Yaoundé I, Yaounde, Cameroon \\ ${ }^{2}$ Yaounde Gynaeco-Obstetric and Pediatric Hospital (YGOPH), Yaounde, Cameroon \\ ${ }^{3}$ Yaounde University Teaching Hospital, Yaounde, Cameroon \\ ${ }^{4}$ Department of Surgery and Sub-Specialties (FMBS), Yaounde, Cameroon \\ Email: *tapouh@gmail.com
}

Received 8 September 2015; accepted 14 December 2015; published 17 December 2015

Copyright (C) 2015 by authors and Scientific Research Publishing Inc.

This work is licensed under the Creative Commons Attribution International License (CC BY).

http://creativecommons.org/licenses/by/4.0/

(c) (i) Open Access

\begin{abstract}
Background: Anterior cruciate ligament (ACL) tears are common complications of knee trauma. This entity can be reliably diagnosed by Magnetic Resonance Imaging. There is a lack of data on the epidemiology of ACL tears in Sub-Saharan Africa. The aim of this study is to describe the radiological aspects of post traumatic ACL tears in a black African setting (Yaounde Teaching Hospital). Methods: ninety six (96) MR studies of the knee were retrospectively reviewed. They were realized on a low field device (0.2T) from July 2012 to December 2013. All the examinations were indicated for knee trauma. Ligamentous, meniscal, bony and joint lesions were sought on coronal and sagittal sections. Results: the sample consists of $70 \%(68 / 96)$ of men. The mean age is $35.36 \pm$ 11.86 years. The prevalence of ACL tears is $45.8 \%(44 / 96)$ of which $36(81.81 \%, 36 / 44)$ are total tears. $23(24 \%)$ have associated meniscal lesion and $8(8.3 \%)$ have a simultaneous damage of the collateral ligaments. The most frequently injured part of the meniscus is its posterior horn $(12 / 23$ cases). Meniscal and "bi-collateral" ligament injuries are independently associated to ACL tears. Conclusion: The prevalence of ACL tears at the Yaounde Teaching Hospital is 45.8\%. It is independently associated to meniscal tears and concomitant injury of the tibial and fibular collateral ligaments.
\end{abstract}

\footnotetext{
${ }^{*}$ Corresponding author.
} 
Keywords

Anterior Cruciate Ligament, Magnetic Resonance Imaging, Meniscal Tears, Knee, Trauma

\section{Introduction}

Anterior cruciate ligament (ACL) tear is one of the commonest ligamentous injuries of the knee [1]. Although its overall incidence in the general population is unknown, there are some estimates suggesting that about 15,000 ACL tears occur due to ski practice in France and 175,000 ACL repairs are performed in United States each year [2]. Sport is the largest provider of ACL tears. In France, five sports are responsible for $90 \%$ of ACL tears: football, skiing, rugby, basketball and judo [3].

A complete physical examination can diagnose ACL tear in $80 \%$ of cases but it is often difficult to achieve in emergency because of pain and it provides little information about the exact extent of injury. That is why medical imaging examinations are usually necessary [4].

Magnetic Resonance Imaging (MRI) is a precious diagnostic tool in a traumatic setting because of its excellent tissue contrast and non-invasive character [5]-[8]. MRI of the knee quickly becomes the largest non-neurological application of this technology and remains the best exploration technique of ACL [9] [10]. With a sensitivity of $90 \%-100 \%$ and a specificity of $82 \%-100 \%$ for the diagnosis of ACL tears, MRI is currently the imaging technique of choice to confirm the suspected diagnosis of rupture and search for associated injuries which may influence the therapeutic protocol [11]-[16].

Despite its many advantages, MRI is a poorly expanded technology in African countries. The first MRI unit in Cameroon was installed at the Yaounde University Teaching Hospital in 2008, followed by another one in Douala city in 2010.

There are numerous patients with knee trauma and suspected ACL tear in our country, as well as qualified orthopaedic surgeons; but to the best of our knowledge, there are no data in the medicalliterature about ACL tears in our setting. Yet the knowledge of the epidemiology of this disease is useful to better organize its prevention and care in a given environment. We, therefore, carry out this study to describe the MRI aspects of ACL tears and its associated lesions in the Yaounde Teaching Hospital (Cameroon).

\section{Methods}

\subsection{Patients and Sampling}

A retrospective review of all patients who underwent a MRI examination of the knee for trauma at the Yaounde Teaching Hospital between July 2012 and December 2013 was performed. This is a national referral hospital with over 40,000 new patients seen per year. Our sample consisted of all patients who came to do an MRI because of knee injury during the study period. Then we excluded those with tumors, infections, gonarthrosis, and previous knee surgery or isolated posterior cruciate ligament tear (Figure 1). Clinical data collected from the electronic medical records were age, sex and the knee's side. The radiological files were reviewed collegially.

\subsection{MRI Protocols and Reading Methods}

The MRI examinations were performed using a $0.2 \mathrm{~T}$ device (Signa Profile Excite Lightspeed VCT, GE Healthcare). Patients were installed supine in the MRI unit with the knee positioned in neutral rotation and extension into a dedicated cylindrical extremity coil. Feet were introduced into the magnet first.

Fast spin echo and proton density T1-weighed and T2-weighed images obtained in sagittal and coronal views were used in this study. Protocols settings were as follows: field of view $18 \mathrm{~cm}$, slice thickness of $3 \mathrm{~mm}$ without interslice gap and a matrix of $512 \times 512$.

Radiological files were stored in DICOM format, transferred to an off-line workstation, and loaded to a software package for image data analysis and visualization (Clear Canvas Workstation 2.0 SP1). Proofreading was done collegially by the main investigator and two senior radiologists on a 21 inch monitor with a maximal resolution of $1368 \times 768$. 


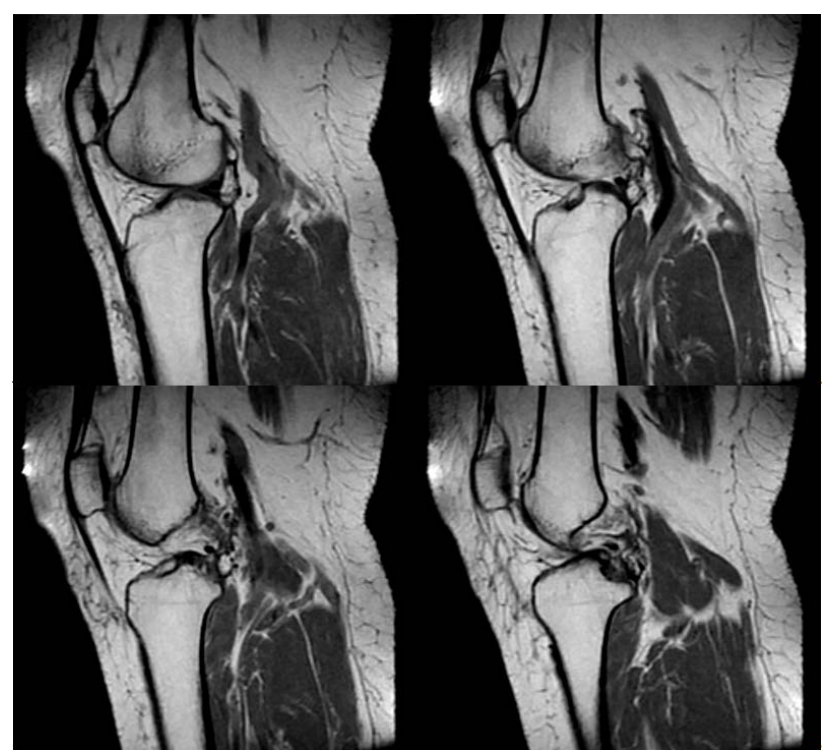

Figure 1. Consecutive sagital views of the knee; proton density fast spin echo weight sequences: total rupture of Anterior Cruciate Ligament and Posterior Cruciate Ligament.

\subsection{Radiological Data of Interest}

ACL tears were classified as total or partial ruptures [3]. Meniscal lesions were searched according to the criteria proposed by Crues and Stoller [17]. As recommended by Ravey et al. we only reported stage III lesions as post traumatic meniscal tears [12]. Other findings sought were: collateral ligament injuries (contusions or rupture), posterior cruciate ligament tears, bone lesions, quadricipital and patellar ligament lesions, anterior tibial translation and joint effusion.

\subsection{Ethical Considerations}

We obtained ethics approval from the ethics committee of the Yaounde Teaching Hospital. It was considered that this study did not require informed consent from patients.

\subsection{Statistical Analysis}

Statistical testing included the Kolmogorov-Smirnov test for normality, Fisher test for testing gender-related variability and logistic regression for assessing association between ACL tears and other lesions. All statistical analyses were performed using SPSS software (SPSS, version 20.0; SPSS Inc., Chicago, IL., USA). The level of statistical significance was set at $p<0.05$.

\section{Results}

\subsection{General Characteristics of the Study Population}

The study population consisted of 96 patients aged 16 to 59 years. The sample was normally distributed ( $p=$ 0.213 ) with a mean age of $35.36 \pm 11.86$ years. There were 68 men (70.8\%; sex ratio: 2.43$)$. The left and right knees were represented in equal numbers $(n=48)$ (Table 1$)$. The overall prevalence of ACL tears was $45.8 \%$ (44/96) with no gender difference $(p=0.20)$. Total tears were strongly predominant (36/44; 81.8\%).

\subsection{Lesions Associated to ACL Tears (Table 2)}

Twenty-three cases (23/96; 24\%) of meniscal tears were found, including 16 cases (36.4\%) significantly associated with ACL tears $(p=0.002)$. Meniscal tears were more frequent on the medial meniscus $(p=0.008)$ and particularly its posterior horn ( $p=0.023)$. Simultaneous rupture of collateral ligaments ("bi-collateral" tear) was 
Table 1. General characteristics of the study population.

\begin{tabular}{|c|c|c|c|}
\hline & $\begin{array}{l}\text { ACL tear } \\
n=44(\%)\end{array}$ & $\begin{array}{c}\text { No ACL tear } \\
n=52(\%)\end{array}$ & $p$-value \\
\hline \multicolumn{4}{|l|}{ Sex } \\
\hline Men & $34(77.3)$ & $34(65.4)$ & 0.20 \\
\hline Women & $10(22.7)$ & $18(34.6)$ & \\
\hline \multicolumn{4}{|l|}{ Age group } \\
\hline 16 to24 years & $11(25)$ & 15 (28.85) & \\
\hline 25 to34 years & $13(29.55)$ & $8(15.38)$ & 0.37 \\
\hline 35 to 44 years & $8(8.18)$ & $14(26.92)$ & \\
\hline 45 to 59 years & $12(27.27)$ & 15 (28.85) & \\
\hline \multicolumn{4}{|l|}{ Kneeinjured } \\
\hline Left & $24(54.5)$ & $24(46.2)$ & 0.41 \\
\hline Right & $20(45.5)$ & $28(53.8)$ & \\
\hline
\end{tabular}

Table 2. ACL tears-associated lesions.

\begin{tabular}{|c|c|c|c|}
\hline & $\begin{array}{l}\text { ACL tear } \\
n=44(\%)\end{array}$ & $\begin{array}{c}\text { No ACL tear } \\
n=52(\%)\end{array}$ & $p$-value \\
\hline Meniscaltear & $17(38.6)$ & $6(11.5)$ & $p=0.002$ \\
\hline \multicolumn{4}{|l|}{ Meniscalsideteared } \\
\hline Medial & $10(22.7)$ & $4(7.7)$ & \\
\hline Lateral & $3(6.8)$ & 0 & $p=0.008$ \\
\hline Medial and lateral & $4(9.1)$ & $2(3.8)$ & \\
\hline \multicolumn{4}{|l|}{ Meniscalhornteared } \\
\hline MM-anteriorhorn & $2(4.5)$ & 0 & $p=0.023$ \\
\hline MM-posteriorhorn & $12(27.3)$ & $5(9.6)$ & \\
\hline LM-anteriorhorn & $4(9.1)$ & $2(3.8)$ & \\
\hline LM-posteriorhorn & $3(6.8)$ & 0 & \\
\hline \multicolumn{4}{|l|}{ Collateral ligament tears } \\
\hline Tibial & $12(27.3)$ & $6(11.5)$ & $p=0.07$ \\
\hline Fibular & $11(25)$ & $5(9.6)$ & $p=0.07$ \\
\hline Tibial and fibular & 7 (15.9) & $1(1.9)$ & $p=0.01$ \\
\hline Meniscaltear & $17(38.6)$ & $6(11.5)$ & $p=0.002$ \\
\hline Meniscalsideteared & & & \\
\hline
\end{tabular}

MM: Medial Meniscus; LM: Lateral Meniscus.

significantly associated with ACL tears ( $p=0.016)$. Joint effusion (49/96, 51.04\%; $p=0.82)$ and bone contusion (29/96, 30.2; $p=0.3$ ) were other frequent joint lesions encountered, but none of them was significantly associated to ACL tears (Table 3).

On multivariate analysis, meniscal tears and "bi-collateral" ligamentous tear were found to be independently associated with ACL tears (Table 4).

\section{Discussion}

This study indicates that ACL tears are the most frequent lesions of the injured knee in Yaounde University Teaching Hospital with $45.8 \%$ prevalence and no gender difference. The factors associated with those lesions are meniscal tears and "bi-collateral" ligamentous tears. The posterior horn of medial meniscus is the most frequently injured. 
Table 3. Miscellaneous joint lesions.

\begin{tabular}{|c|c|c|c|}
\hline & $\begin{array}{l}\text { ACL tear } \\
n=44(\%)\end{array}$ & $\begin{array}{c}\text { No ACL tear } \\
n=52(\%)\end{array}$ & $p$-value \\
\hline Joint effusion & $23(52.3)$ & $26(50)$ & 0.82 \\
\hline \multicolumn{4}{|l|}{ Contusion of tibial condyles } \\
\hline Lateral & $4(9.1)$ & $3(5.8)$ & 0.26 \\
\hline Medial & $6(13.6)$ & $3(5.8)$ & \\
\hline \multicolumn{4}{|l|}{ Contusion of femoral condyles } \\
\hline Lateral & $6(13.6)$ & $1(1.9)$ & 0.35 \\
\hline Medial & $4(9.1)$ & $2(3.8)$ & \\
\hline Elongation of the patellar tendon & $3(6.8)$ & $1(1.9)$ & 0.4 \\
\hline Posterior cruciate ligament tear & $3(6.8)$ & 0 & 0.96 \\
\hline Anterior tibial translation & $2(4.6)$ & 0 & 0.20 \\
\hline Contusion of the fibular head & $1(2.3)$ & 0 & 0.45 \\
\hline Avulsion of the intercondylar tubercles & $1(2.3)$ & 0 & 0.45 \\
\hline
\end{tabular}

MM: Medial Meniscus; LM: Lateral Meniscus.

Table 4. Lesions associated to ACL tears on multivariate analysis.

\begin{tabular}{ccc} 
Lesion & $p$-value & $\beta^{*}[95 \%$ IC] \\
Meniscal tears & 0.004 & $4.74[1.63-13.82]$ \\
"Bi-collateral” ligamentous tear & 0.04 & $9.33[1.05-28.83]$ \\
\hline
\end{tabular}

${ }^{*} \beta$ : Logistic regression coefficient.

\subsection{Prevalence of ACL Tears}

The predominance of ACL tears in traumatic knee disorders is now widely accepted [3] [18]. ACL is the main obstacle to anterior tibial translation; it provides secondary stabilization in response to internal tibial rotation and varus or valgusangulation. The main mechanisms of knee trauma are: external tibial rotation, internal rotation of the femur, extreme valgus unintentionally applied to a flexed knee, hyperextension and valgus or direct side impact [2].

\subsection{Association with Collateral Ligamentous Tears}

The association between ACL tears and "bi-collateral” tears had not been previously reported. The tibial collateral ligament is more often injured in case of knee trauma due to forced valgus which causes its stretching and tearing, meanwhile the fibular collateral ligament can be broken due to external rotation or direct lateral knee injury [1] [13]. According to Guenoun et al. forced valgus and external rotation are part of the most common ACL tears mechanism, which may justify their association with "bi-collateral” ligamentous tears [3].

\subsection{Association with Meniscal Tears}

The association between ACL tears and meniscal tears is well established in the literature as stated by Lecouvet et al. [1]. The medial meniscus is usually more injured because it is thinner, narrower and more "opened” than the lateral meniscus [8]. Lerais et al. had found that the posterior horn is preferably teared because meniscal tears often start there and gradually extend to the front, so that isolated lesions of the anterior horn of the medial meniscus does not exist [19].

\subsection{Gender Influence}

We did not find any gender difference in the prevalence of ACL tears which is not consistent with the data of li- 
terature recalled by Cimino et al. who stated that there is 1.4 to 9.5 times increased risk of ACL tears in women [4]. Some theories for this predominance in women have been suggested: poorer access to training facilities, hamstring strength, hormonal fluctuations, narrow intercondylar notch, increased $Q$ angle and quadriceps dominance [4] [20] [21]. The absence of gender difference in our study is probably due to the overrepresentation of men who constituted $70.8 \%$ of the sample. Men are probably more often the victims of knee injury than women because they are most likely to practice sport which is the first provider of ACL tears [22].

\subsection{Limitations to the Study}

We performed our examinations with a low field magnet $(0.2 \mathrm{~T})$. Several studies comparing low field $(0.2 \mathrm{~T})$ and high field (above $1.5 \mathrm{~T}$ ) devices in the evaluation of ACL tears have found a poorer image quality and a longer examination time with low fields despite a comparable diagnostic accuracy [23] [24]. Thus, we considered this was not a limitation to carry out the study.

Three real limitations to this study should be mentioned: the limited sample size, overrepresentation of men and the lack of clinical data. MRI is a recent and still expensive technology (at least 225 U.S. dollars) in our country; clinicians do not prescribe enough and there are few patients who can afford it. The retrospective design of this study did not allow us to collect more clinical data in order to integrate them into our analysis and verify if, as previously reported, they could improve the detectability of ACL tears [5]. There is a necessity to continue this study prospectively on a larger and balanced sample in terms of gender, in order to validate its findings.

\subsection{Suggestions}

Our results suggest that in case of knee trauma, the presence of a "bi-collateral" ligamentous tear (recognizable on clinical or ultrasound examination) increases the probability of ACL tear. This could be very useful in our country where MRI is not yet attainable for the majority of the population.

\section{Conclusion}

ACL tears are the most frequent lesions of the injured knee in Yaounde University Teaching Hospital and they are commonly total tears. Their prevalence is $45.8 \%$ without gender difference. The factors associated with those lesions are meniscal tears and "bi-collateral" ligamentous tears. The posterior horn of medial meniscus is the most frequently injured. Further prospective and population-based studies will be necessary to assess this particular association and provide more generalizable results.

\section{References}

[1] Lecouvet, F., Malghem, J. and Poilvache, P. (2004) Berg BV: 2108 Genou: Imagerie des lesions ligamentaires, des ligamentoplastieset de leurs complications. Journal de Radiologie, 85, 1179. http://dx.doi.org/10.1016/S0221-0363(04)76564-6

[2] Kam, C.K., Chee, D.W.Y. and Peh, W.C.G. (2010) Magnetic Resonance Imaging of Cruciate Ligament Injuries of the Knee. Canadian Association of Radiologists Journal, 61, 80-89. http://dx.doi.org/10.1016/j.carj.2009.11.003

[3] Guenoun, D., Le Corroller, T., Amous, Z., Pauly, V., Sbihi, A. and Champsaur, P. (2012) The Contribution of MRI to the Diagnosis of Traumatic Tears of the Anterior Cruciate Ligament. Diagnostic and Interventional Imaging, 93, 331341. http://dx.doi.org/10.1016/j.diii.2012.02.003

[4] Cimino, F., Volk, B.S. and Setter, D. (2010) Anterior Cruciate Ligament Injury: Diagnosis, Management, and Prevention. American Family Physician, 82, 917-922.

[5] Dejour, D., Ntagiopoulos, P.G., Saggin, P.R. and Panisset, J.C. (2013) The Diagnostic Value of Clinical Tests, Magnetic Resonance Imaging, and Instrumented Laxity in the Differentiation of Complete versus Partial Anterior Cruciate Ligament Tears. Arthroscopy, 29, 491-499. http://dx.doi.org/10.1016/j.arthro.2012.10.013

[6] Tavernier, T. and Dejour, D. (2001) Knee Imaging: What Is the Best Modality. Journal de Radiologie, 82, 387-388.

[7] Boutry, N., Bourges, M., Dupont, S., Budzik, J., Demondion, X. and Cotton, A. (2009) Value of Imaging in Posterolateral Corner Injuries of the Knee. Journal de Radiologie, 90, 681-691.

http://dx.doi.org/10.1016/S0221-0363(09)74723-7 
[8] Shahabpour, M., DeMeyere, N., DeMaeseneer, M., David, P., DeRidder, F. and Stadnik, T. (2005) Anatomienormale du genou en imagerie par résonancemagnétique. EMC-Radiologie, 2, 165-182.

http://dx.doi.org/10.1016/j.emcrad.2004.12.001

[9] Burk Jr., D.L., Mitchell, D.G., Rifkin, M.D. and Vinitski, S. (1990) Recent Advances in Magnetic Resonance Imaging of the Knee. Radiologic Clinics of North America, 28, 379-393.

[10] Drape, J.L., Godefroy, D., Dupont, A.M. and Chevrot, A. (1999) Radio-Anatomy of the Knee. Journal de Radiologie, 80, 628-639.

[11] Laoruengthana, A. and Jarusriwanna, A. (2012) Sensitivity and Specificity of Magnetic Resonance Imaging for Knee Injury and Clinical Application for the Naresuan University Hospital. Journal of the Medical Association of Thailand, 95, S151-S157.

[12] Ravey, J.N., Pittet-Barbier, L. and Coulomb, M. (2004) Imagerie par résonance magnétique des lésions méniscoligamentaires du genou. EMC—Radiologie, 1, 393-425. http://dx.doi.org/10.1016/j.emcrad.2004.02.003

[13] Bining, J., Andrews, G. and Forster, B.B. (2009) The ABCs of the Anterior Cruciate Ligament: A Primer for Magnetic Resonance Imaging Assessment of the Normal, Injured and Surgically Repaired Anterior Cruciate Ligament. British Journal of Sports Medicine, 43, 856-862. http://dx.doi.org/10.1136/bjsm.2008.050492

[14] Klass, D., Toms, A.P., Greenwood, R. and Hopgood, P. (2007) MR Imaging of Acute Anterior Cruciate Ligament Injuries. The Knee, 14, 339-347. http://dx.doi.org/10.1016/j.knee.2007.04.008

[15] Moore, S.L. (2002) Imaging the Anterior Cruciate Ligament. Orthopedic Clinics of North America, 33, 663-674. http://dx.doi.org/10.1016/S0030-5898(02)00022-6

[16] Ho, C.P., Marks, P.H. and Steadman, J.R. (1999) MR Imaging of Knee Anterior Cruciate Ligament and Associated Injuries in Skiers. Magnetic Resonance Imaging Clinics of North America, 7, 117-130.

[17] Stoller, D.W., Martin, C., Crues III, J.V., Kaplan, L. and Mink, J.H. (1987) Meniscal Tears: Pathologic Correlation with MR Imaging. Radiology, 163, 731-735. http://dx.doi.org/10.1148/radiology.163.3.3575724

[18] Teske, W., Anastisiadis, A., Lichtinger, T., von Schulze Pellengahr, C., von Engelhardt, L.V. and Theodoridis, T. (2010) Rupture of the Anterior Cruciate Ligament. Diagnostics and Therapy. Orthopade, 39, 883-898; Quiz 899. http://dx.doi.org/10.1007/s00132-010-1670-9

[19] Lerais, J.M., Mercky, N., Régis, A., Loffroy, R., Guiu, B., Runge, M. and Kastler, B. (2008) Apports de l’IRM 3T dans les lesions meniscales du genou. Journal de Radiologie, 89, 1375. http://dx.doi.org/10.1016/S0221-0363(08)76149-3

[20] Sutton, K.M. and Bullock, J.M. (2013) Anterior Cruciate Ligament Rupture: Differences between Males and Females. Journal of the American Academy of Orthopaedic Surgeons, 21, 41-50. http://dx.doi.org/10.5435/JAAOS-21-01-41

[21] Boden, B.P., Sheehan, F.T., Torg, J.S. and Hewett, T.E. (2010) Noncontact Anterior Cruciate Ligament Injuries: Mechanisms and Risk Factors. Journal of the American Academy of Orthopaedic Surgeons, 18, 520-527.

[22] Majewski, M., Susanne, H. and Klaus, S. (2006) Epidemiology of Athletic Knee Injuries: A 10-Year Study. The Knee, 13, 184-188. http://dx.doi.org/10.1016/j.knee.2006.01.005

[23] Cotton, A., Delfaut, E., Demondion, X., Lapegue, F., Boukhelifa, M., Boutry, N., Chastanet, P. and Gougeon, F. (2000) MR Imaging of the Knee at 0.2 and 1.5 T: Correlation with Surgery. American Journal of Roentgenology, 174, 10931097. http://dx.doi.org/10.2214/ajr.174.4.1741093

[24] Vellet, A.D., Lee, D.H., Munk, P.L., Hewett, L., Eliasziw, M., Dunlavy, S., Vidito, L., Fowler, P.J., Miniaci, A. and Amendola, A. (1995) Anterior Cruciate Ligament Tear: Prospective Evaluation of Diagnostic Accuracy of Middle- and High-Field-Strength MR Imaging at 1.5 and 0.5 T. Radiology, 197, 826-830. 